\title{
Approach to a Method of Integrated Evaluation of Thermal Fatigue and its Validation Using SPECTRA*
}

\author{
Toru OUMAYA ${ }^{* *}$, Akira NAKAMURA $^{* *}$ and Nobuyuki TAKENAKA ${ }^{* * *}$ \\ ${ }^{* *}$ Institute of Nuclear Safety System, Inc, \\ 64 Sata Mihama-cho Mikata-gun Fukui-ken 919-1205 Japan \\ E-mail: ooumaya@inss.co.jp \\ ***Kobe University, \\ 1-1 Rokkodai Nada Kobe-Shi, Hyogo-ken 657-8501 Japan \\ E-mail: takenaka@mech.kobe-u.ac.jp
}

\begin{abstract}
Thermal fatigue may initiate at a T-junction or a branched off line where high and low temperature fluids mix. These are common piping elements in nuclear power plants. To ensure structural integrity against thermal fatigue during the design phase, it is important to estimate thermal load from such design specifications as flow rate, temperature difference, pipe diameter, etc. IMAT-F, an evaluation method integrating thermal hydraulic and structural analysis, was developed in this study to precisely determine thermal load excluding safety margins or conservative engineering judgment. The method was validated by numerical flow simulations of high-cycle thermal fatigue experiment SPECTRA, conducted by Japan Atomic Energy Agency. Results confirmed that IMAT-F can accurately simulate fluid and pipe wall temperature fluctuation using fluid-structure coupled analysis. Thermal stress fluctuation resulting from distribution of temperature fluctuation in the pipe wall was then calculated. Fluctuation fatigue life was also estimated for comparison with the experimental results.
\end{abstract}

Key words: Thermal Fatigue, Thermal Stress, Temperature Fluctuation, Fluid-Structure Coupled Analysis

\section{Introduction}

There have been several instances of leakage due to thermal fatigue cracks in nuclear power plants. Some involved complex flow phenomena at point of hot and cold flow mix. In 2003, the Japan Society of Mechanical Engineers published "Guideline for Evaluation of High-Cycle Thermal Fatigue of a Pipe"(1) to establish and prescribe design process methodology to prevent thermal fatigue cracks. The guideline targets thermal striping at mixing tees and thermal stratification at closed-end branch pipes. The guideline prescribes an evaluation flow chart consisting of four separate steps, with an option for detailed evaluation in the final steps, when the cumulative usage factor exceeds one applying all four steps. One example of this detailed evaluation comprises numerical simulation of local flow at a specific location, based on design conditions. Also, for root cause analysis of suspected thermal fatigue leakage, detailed structural analysis is recommended, estimating precise thermal load in consideration of local flow phenomena.

The Japan Atomic Energy Agency (JAEA) is currently developing an evaluation method $^{(2)}$ that combines computational fluid dynamics (CFD) with thermal conductive and 
stress analysis., Électricité de France (EDF) ${ }^{(3)}$ and the French Atomic Energy Commission $(\mathrm{CEA})^{(4)}$ both developed original evaluation methods for thermal fatigue, to investigate the root cause of leakage in the residual heat removal system of Civaux Unit No.1 in 1998. These organizations employ originally developed codes for CFD, thermal conductive, and stress analysis.

In the present study, an integrated evaluation method named "Integrated Methodology for Assessing Thermal Fatigue" (IMAT-F) was developed, which employs widely used general-purpose commercial code and mock-up experiment output.

To validate the IMAT-F method, which applies numerical flow simulation outputs, a

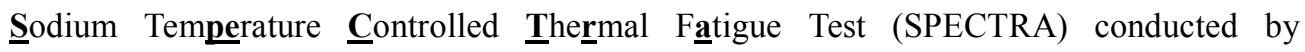
JAEA ${ }^{(5)(6)}$ was simulated. SPECTRA loop was intended to generate temperature fluctuation of liquid sodium in a T-junction of the test section and initiate cracks at the inner surface of the test section. Since the Plandtl number of liquid sodium is smaller than that of water, the experiment's CFD simulation output is less affected by the computational meshing near the wall. Thus, this experiment is considered suitable for IMAT-F method validation.

This paper introduces the basic concept of the IMAT-F method which applies numerical flow simulation, and its validation with SPECTRA. To demonstrate advantages of the integrated method, the effect of the turbulence model on thermal stress is also compared with three types of models.

\section{The basic concept of thermal fatigue systematic evaluation}

IMAT-F is outlined in the block diagram in Fig. 1. In IMAT-F, general-purpose commercial codes were employed for CFD and structural analysis. These codes have been employed in many fields, including those of thermal fatigue, so it was considered beneficial to incorporate their progress. This study also employed CFX for CFD analysis and ABAQUS for structural analysis.

The main features of IMAT-F are that it automatically links CFD analysis and experimental outputs with structural analysis inputs by processing data conversion and interpolation, and calculates maximum stress amplitude and cumulative usage factor for all nodes while determining which node has maximum value. These values must be calculated for all regional nodes since it is difficult to predict the amount and location of the maximum usage factor, due to flow phenomena local and random characteristics. The main features are shown in the block diagram in Fig. 2, along with the relation of each feature. Three separate methods are integrated in IMAT-F.

"Method \#1" uses experimental output: fluid local temperature fluctuation is obtained by the experiment. The adequate heat transfer coefficient best suit with the case must then be determined by the user. In order to precisely determine thermal stress using "Method \#1", the unsteady heat transfer coefficient should be determined for the particular flow phenomenon. Obtaining the coefficient necessitates a thermal hydraulic mock-up experiment in which the pipe temperature at the inner wall and fluid temperature near the wall are measured simultaneously, and the heat flux for the particular experiment condition is calculated. In practical application, "Method \#1" is intended to survey the effect of heat transfer coefficient when applying the commonly used Dittus-Boelter correlation as reference, and comparing the result with those of other methods. The Dittus-Boelter correlation is commonly used in heat transfer analysis during the design phase, since it is been widely known to agree relatively well for steady turbulent flow in a pipe.

"Method \#2" also uses experimental output: temperature fluctuations of the pipe's inner wall are obtained by mock-up experiment. Since the number of measurement points is limited, interpolation should be processed to obtain the two-dimensional temperature distribution fields required for structural analysis. IMAT-F processes this interpolation 
automatically and converts experimental output into boundary condition for structural analysis.

"Method \#3" uses numerical simulation output: fluid-structure thermally coupled analysis calculates the pipe wall temperature distribution directly using the CFD code. IMAT-F automatically converts CFD analysis output to input data for structural analysis. Since IMAT-F can distinguish structural domain and fluid domain, nodes for CFD and structural analysis can be different. In other words, a coarsely meshed model is allowed for structural analysis in IMAT-F.

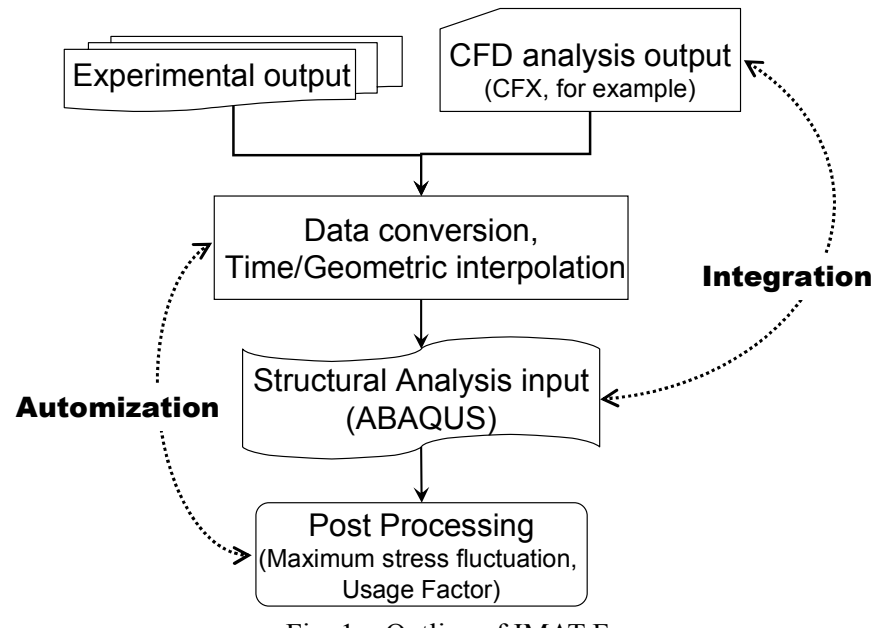

Fig. 1 Outline of IMAT-F

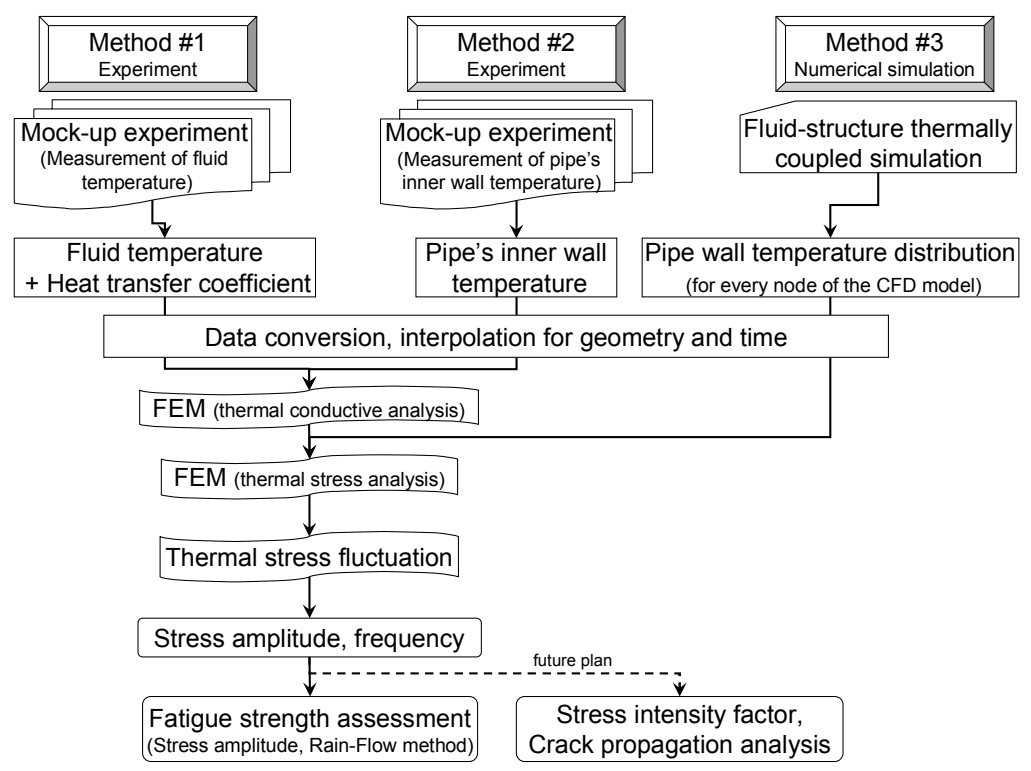

Fig. 2 Main features of IMAT-F

\section{Validation of numerical simulation}

\subsection{SPECTRA Experiment ${ }^{(5)(6)}$}

To validate the "Method \#3" shown in Fig. 2, the experiment SPECTRA conducted by JAEA was used. The SPECTRA loop is outlined in Fig. 3. A SPECTRA loop can generate sinusoidal temperature fluctuation of sodium with constant flow velocity. The high (525 deg C) and low (325 deg C) temperature liquid sodium flows alternately into the test section. A detailed diagram of the test section is shown in Fig. 4. The test section is made of 304 type 
stainless steel, with pipe thickness $4.7 \mathrm{~mm}$ at test section inlet and $11.1 \mathrm{~mm}$ at outlet. Downstream of the porous plate, the so-called 'mixing region' was thickened to produce more temperature gradient along the radial direction. At the T-junction, a thermal sleeve was attached to protect the pipe wall. Experimental output with 20-second period cycle and temperature range of $200 \mathrm{deg} \mathrm{C}$ was chosen for numerical simulation. In the experiment, cracks penetrated the pipe wall in 157,150 cycles $^{(5)}$, about 36 days. Three through-wall cracks were noted along the axial direction, and many shallow cracks were observed near the tapered region of the test section. Striation marks were observed on the surfaces of cracks.

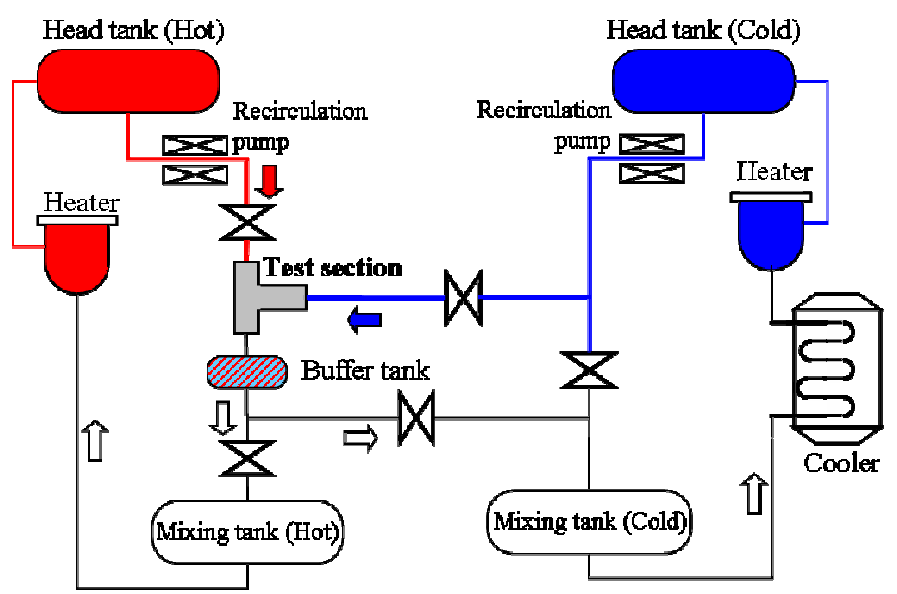

Fig. 3 SPECTRA test loop ${ }^{(5)}$

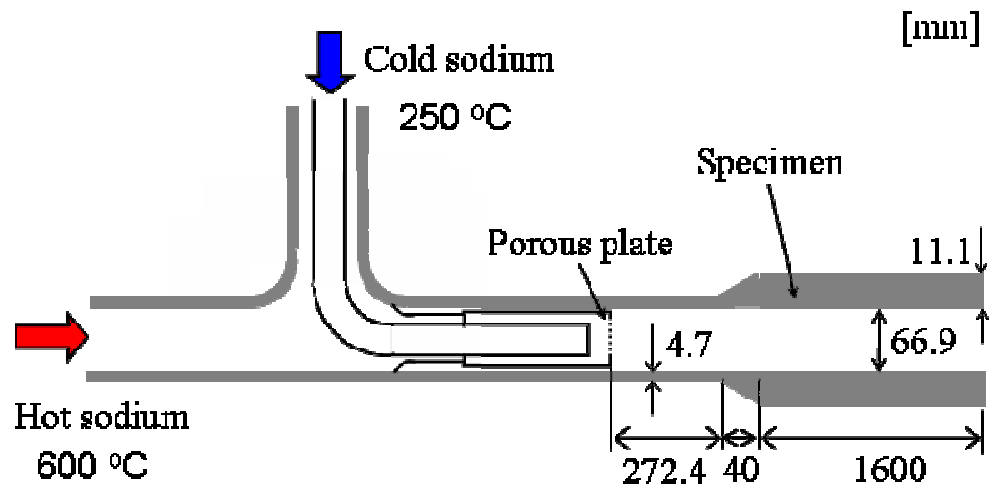

Fig. 4 Detailed diagram of test section ${ }^{(5)}$

\subsection{Numerical conditions for CFD analysis}

The general purpose commercial code $\mathrm{CFX}-10^{(7)}$ developed by ANSYS Inc. was employed for CFD analysis in this simulation. CFX-10 can calculate a fluid-structure thermally coupled model, that is, it can simulate fluid temperature and pipe-wall temperature simultaneously. Thermal convection was simulated accordingly, with flow condition and wall function of the turbulence model, without assuming any constant experimental correlation for heat transfer between fluid and pipe inner wall.

\subsubsection{Simulated conditions}

The flow conditions at the porous plate inlet were set as constant during the experiment: mean velocity was $\bar{U}=1.42 \mathrm{~m} / \mathrm{s}$ and temperature fluctuation was in the range of 325 to 525 degrees Centigrade in a sinusoidal manner. The material properties of liquid sodium, 
such as viscosity, density, heat capacity and thermal conductivity, were set as temperature-dependent variables. Buoyancy was neglected in the analysis. Initial temperature was 325 degrees Centigrade for both fluid and pipe wall.

Three types of turbulence models were selected for result comparison with IMAT-F: k- $\varepsilon$ model, Shear Stress Transport k- $\omega$ (SST) model and Detached Eddy Simulation (DES). In the simulation, a second-order upwind scheme was used for the k- $\varepsilon$ and SST models and a second-order central scheme was used for the DES model, as difference schemes of the advection term. A second-order Euler implicit scheme was used as a difference scheme of the time for all cases.

\subsubsection{Modeling}

The test section was divided into fluid and structural domains in the computational mesh of CFX-10, for simulation with the fluid-structure thermally coupled model.

Model meshing was conducted accordingly, with the following.

\section{1) Simulated area}

There was a perforated plate at the mixing region inlet to accelerate diffusion and obtain proper sinusoidal temperature fluctuation. In the simulation, the test section was modeled from the perforated plate to the tapered region of the pipe for axial direction. Since circumferential symmetry could be achieved with $1 / 6^{\text {th }}$ for the perforated plate, only $1 / 6^{\text {th }}$ of the cross-section was modeled for the circumferential direction.

2) Computational mesh

The cross-section of the computational mesh with the turbulence models of k- $\varepsilon$ and SST is shown in Fig. 5(a); that with DES is shown in Fig. 5(b). The entire turbulence model computational mesh is outlined in Fig. 5(c). The total number of node was 224,930 for the $\mathrm{k}-\varepsilon$ and SST models (153,965 nodes in the fluid domain and 70,965 nodes in the structural domain), and 547,302 for DES (367,524 nodes in the fluid domain and 179,778 nodes in the structural domain). Non-dimensional distance $\mathrm{y}+$ was set at 50 for the $\mathrm{k}-\varepsilon$ and SST models, and at 20 for the DES model to accommodate the meshing guidelines for $\mathrm{CFX}^{(7)}$, which recommend that $\mathrm{y}+$ be from $20 \sim 100$. The thickness of the first mesh in the fluid domain adjacent to the structural domain was set to be $y=0.3$ and $0.1 \mathrm{~mm}$ when $\mathrm{y}+$ was 50 and 20, respectively. The simulated model axial direction mesh width was $\mathrm{z}=5 \mathrm{~mm}$ for all cases.

3) Time increment

The time increment must satisfy the condition expressed in Formula (1) for stable running of thermal conduction simulation ${ }^{(8)}$.

$$
\Delta t<\frac{\rho_{s} c_{s}(\Delta z)^{2}}{2 \lambda_{s}}
$$

where $\rho_{s}$ is the density, $c_{s}$ is the heat capacity and $\lambda_{s}$ is the thermal conductivity of the pipe's material, defined at 425 degrees Centigrade. When the axial direction mesh width is $\Delta z=5 \mathrm{~mm}, \Delta t$ should be less than $0.19 \mathrm{~s}$. Therefore, the time increment was set at $0.05 \mathrm{~s}$ for simulations with the $\mathrm{k}-\varepsilon$ and SST models.

As for the DES model, in addition to Formula (1), it is advised that the Courant number be set less than one. Therefore, the time increment of $\Delta \mathrm{t}<0.0035 \mathrm{~s}$ should be satisfied when the axial flow velocity is $\bar{U}=1.42 \mathrm{~m} / \mathrm{s}$ and $\Delta z=5 \mathrm{~mm}$. The time increment was set at 0.002 $\mathrm{s}$ for simulation with the DES model.

Overall calculation length was set at $100 \mathrm{~s}$, which is five cycles of the temperature fluctuation, for all cases. 


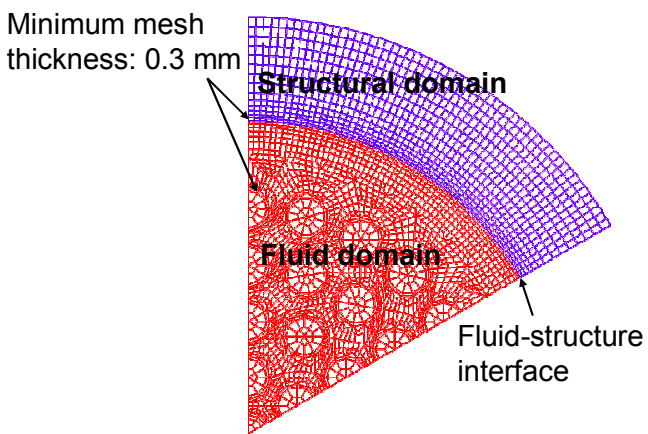

(a) Computational mesh for $\mathrm{k}-\varepsilon$ and SST, cross-section

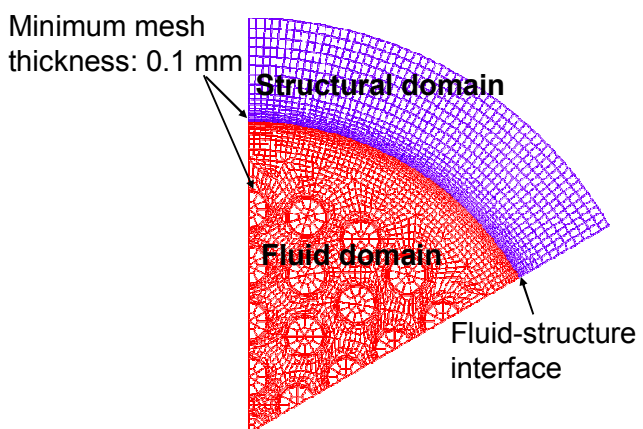

(b) Computational mesh for DES, cross-section

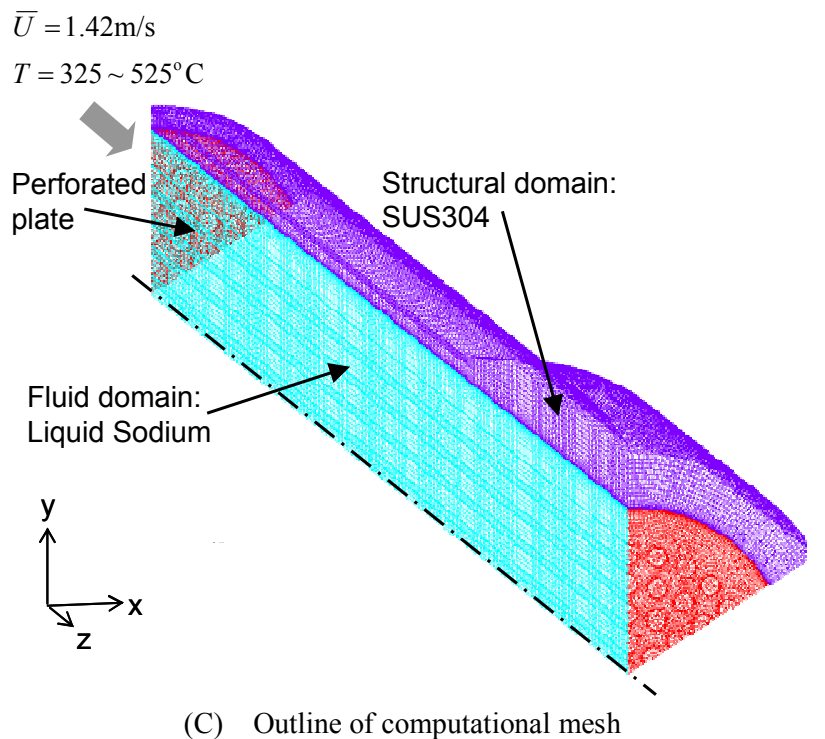

Fig. 5 Computational meshes for CFD analysis

\subsection{Numerical conditions for structural analysis}

The general purpose commercial code ABAQUS $6.5^{(9)}$ developed by ABAQUS Inc. was employed for finite element analysis.

\subsubsection{Modeling}

The computational mesh for structural analysis is outlined in Fig. 6. The test section was modeled for $1 / 6^{\text {th }}$ of the cross-section, for consistency with the CFD analysis model. The 20 node second-order three-dimensional solid element was employed for the analysis. The total number of nodes was 35,341 . The number of nodes was significantly smaller and 
their coordinates were not consistent with the structural domain of the CFD analysis model, so as to demonstrate the data-conversion capabilities of IMAT-F. Both inlet and outlet displaced as rigid flat surfaces, with no displacement allowed at the inlet center and only axial displacement allowed at the outlet center. Young's modulus, the coefficient of thermal expansion $^{(10)}$ and thermal conductivity ${ }^{(11)}$ were set as temperature dependent variables.

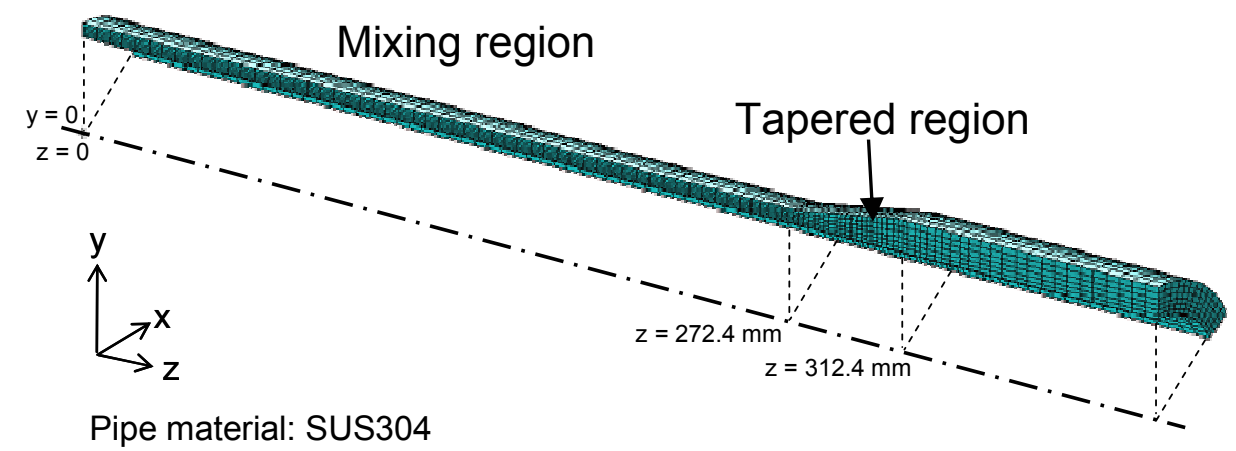

Fig. 6 Computational mesh for structural analysis

\section{Results of Numerical Simulation}

\subsection{Flow velocity}

The instantaneous distribution of the velocity vector simulated with the DES model when $t=40 \mathrm{~s}$ is shown in Fig. 7. Jet flow heading toward the mixing region is observed at the perforated plate outlet. In the area surrounded by the outermost holes of the perforated plate and the pipe's inner wall, counterflow is observed from $z=0$ to $60 \mathrm{~mm}$, due to recirculation caused by the jet flow. As the jet flows downstream, the radial velocity distribution gradually approaches a mean velocity of $\bar{U}=1.42 \mathrm{~m} / \mathrm{s}$. Similar distribution was observed in the results with the k- $\varepsilon$ and SST models. Since the velocity vector instantaneous distribution did not fluctuate over time, the flow in the mixing region was considered to be in a steady state for all cases.

The instantaneous velocity distribution for the radial (y) direction is shown in Fig. 8 for the three different turbulence models. The distribution at $z=50 \mathrm{~mm}$ (within the recirculation zone) and $372.4 \mathrm{~mm}$ (tapered region outlet) is shown in Fig. 8 (a) and (b), respectively. Multiple peaks were observed corresponding to the holes in the perforated plate for the result with the DES model, shown in Fig. 8(a). Since computational meshing around the holes in the perforated plate was similar to the other turbulence models, the DES model must have calculated the velocity peak as stronger and the jet flow diffusion as weaker than the other turbulence models. Planar velocity distribution was observed downstream, where $z=372.4 \mathrm{~mm}$ and comparison between the turbulence models indicates that velocity peak remains strong at the center in the result for the DES model. 


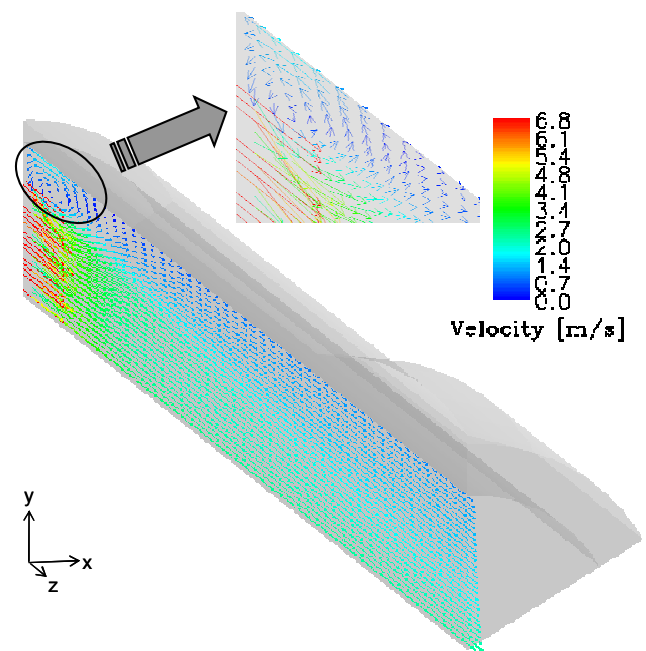

Fig. 7 Velocity vector distribution of mixing region, DES at $t=40 \mathrm{~s}$

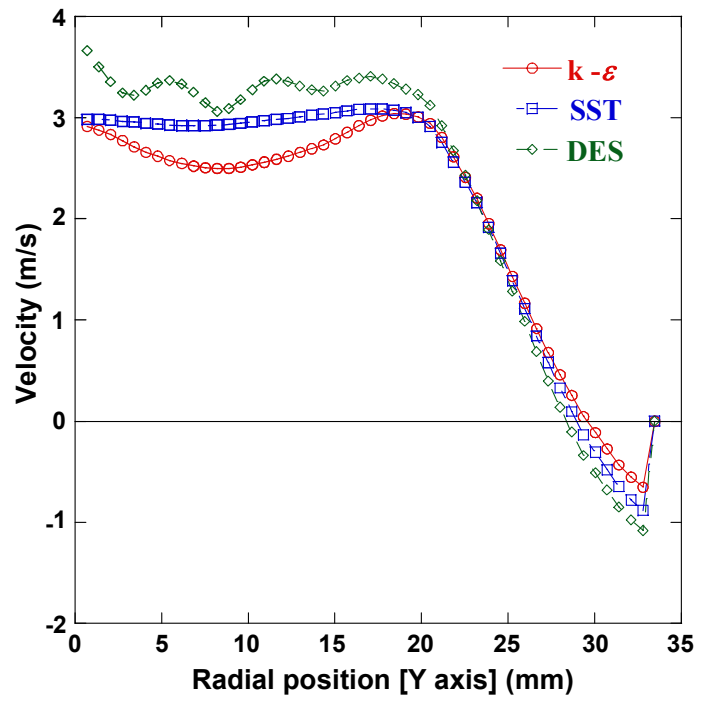

(a) $z=50 \mathrm{~mm}$

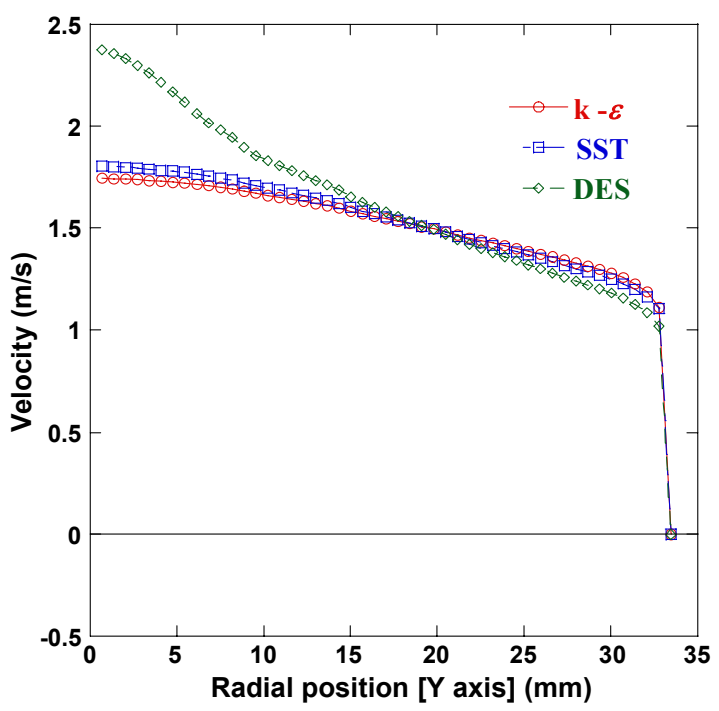

(b) $z=372.4 \mathrm{~mm}$

Fig. 8 Velocity distribution for radial direction, $t=5 \mathrm{~s}$ 


\subsection{Simulated temperature}

\subsubsection{Temperature distribution}

The instantaneous temperature distribution of the mixing region at maximum and minimum times ( $t=10$ and 20, respectively), as simulated with the DES model, is shown in Fig. 9. When the fluid temperature was maximum, the pipe wall temperature was lower than that of the fluid. In addition, the pipe wall temperature at the tapered region outlet was lower than at the inlet. This indicates that as the fluid temperature fluctuation is conducted through the pipe wall, the phase of fluctuation delays more at the tapered region outlet, due to greater thermal capacity within the pipe wall.

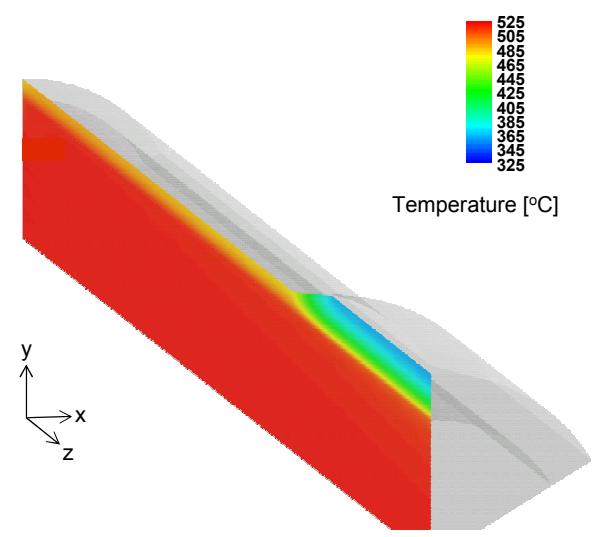

(a) $t=10 \mathrm{~s}$ (Maximum fluid temperature)

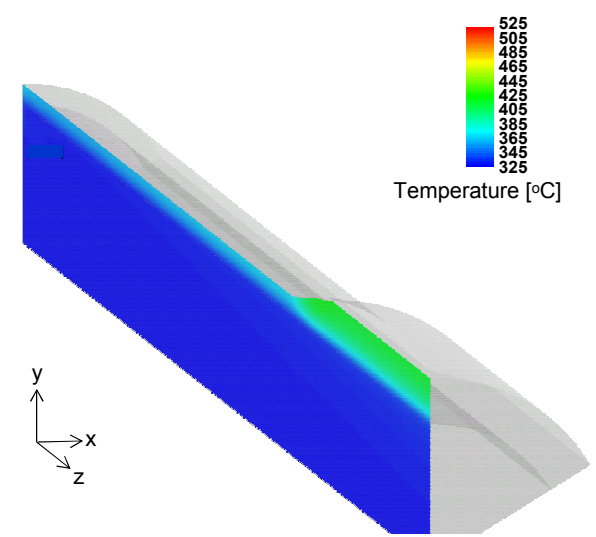

(b) $t=20 \mathrm{~s}$ (Minimum fluid temperature)

Fig. 9 Instantaneous temperature distribution of test section (DES)

\subsubsection{Temperature fluctuation}

The temperature fluctuations of fluid and pipe wall as simulated with the DES model at the tapered region outlet $(z=372.4 \mathrm{~mm})$ are shown in Fig. 10. The temperature fluctuations in the experiment conducted by Hasebe et al. ${ }^{(6)}$ are also plotted in Fig. 10. Three cycles of simulated fluctuation were extracted from the overall result, and the phases were adjusted to fit the measurement. The fluid temperature fluctuations showed good agreement with the experimental results, as shown in Fig. 10(a). The pipe wall temperature fluctuations were also in good agreement with the measurement, as shown in Fig. 10(b). Experimental results had a smaller phase delay and temperature fluctuation amplitudes were slightly larger.

The radial temperature distribution of simulated results using the DES model and measurements taken when fluctuation at the inner wall reached maximum $(47.5 \mathrm{~s})$ and minimum (37.45s) points are shown in Fig. 11. Also shown is the temperature distribution $T_{r}$ calculated based on theoretical maximum temperature amplitude. It was calculated using the following formula ${ }^{(12)}$. 


$$
\begin{aligned}
& \operatorname{Tr}=\frac{2 h^{*}}{\sqrt{\left(h^{*}+k\right)^{2}+k^{2}}} e^{-k x}+425 \\
& h^{*}=h / \lambda, k=\sqrt{\omega / 2 a}
\end{aligned}
$$

where $h$ is the heat transfer coefficient, $\lambda$ is the thermal conductivity, $a$ is the thermal diffusivity factor, $\omega$ is the angular frequency, and $x$ is the radial position. $h$ is calculated based on heat flux simulated using the DES model and $\lambda$ is defined at 425 degrees Centigrade, which is the average temperature of the fluctuation. The simulated temperature distributions in the radial direction were also in good agreement with the measurement. In comparing the simulated result and measurements with the theoretical value, the difference increases as the radial position approaches the outer surface. Since the phase delay increases as the radial position approaches the outer surface, as shown in Fig. 10(b), divergence from the maximum temperature fluctuations increased.

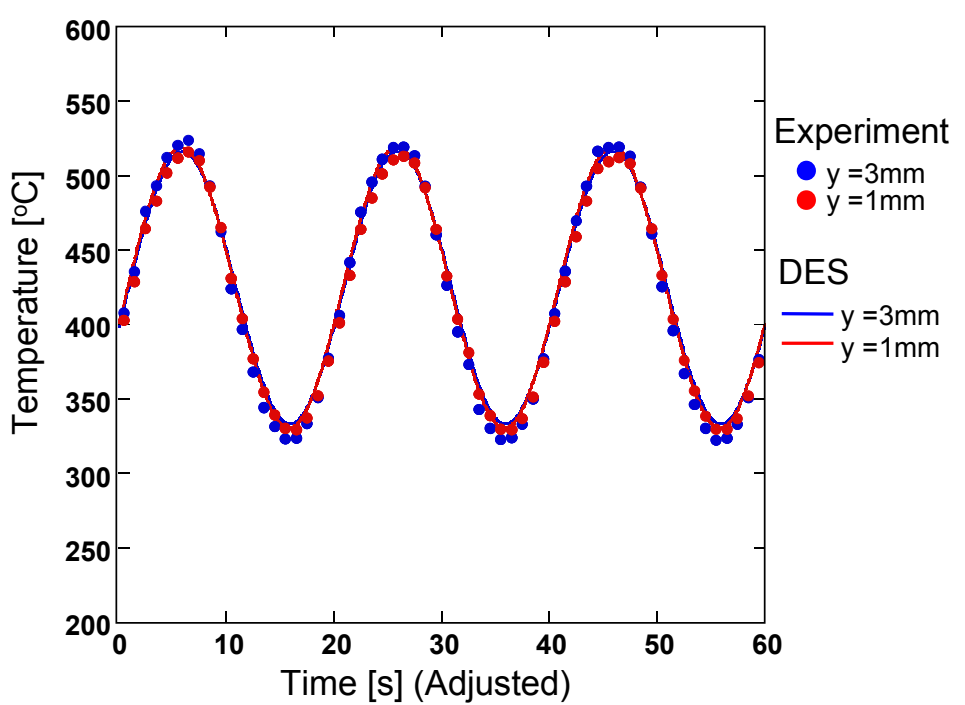

(a) Fluid temperature

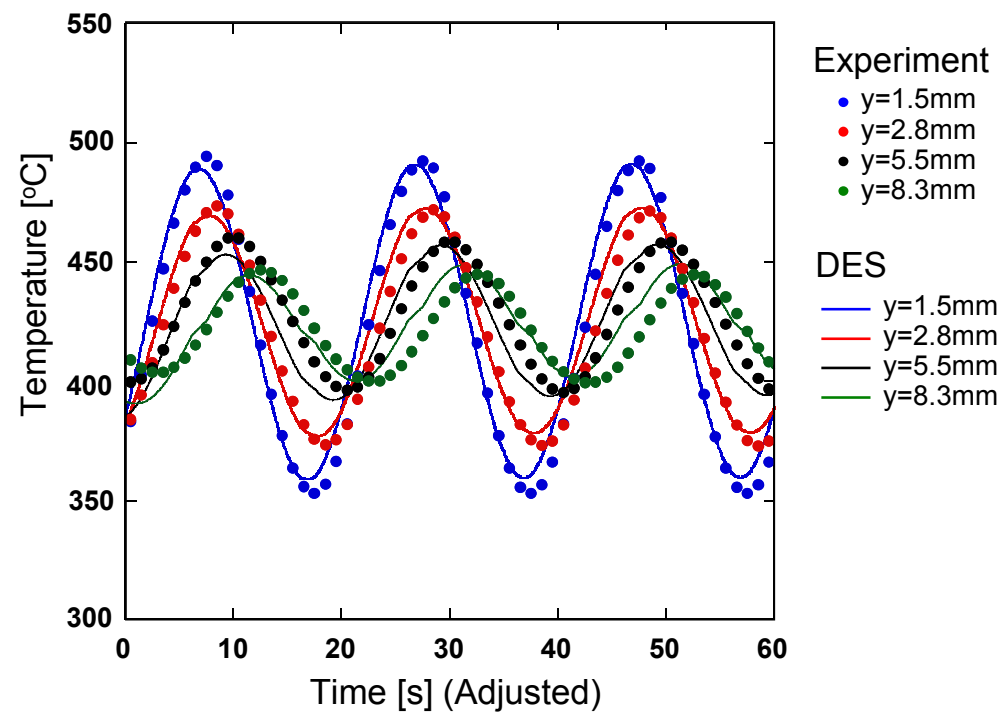

(b) Pipe wall temperature

Fig. 10 Temperature fluctuations of fluid and pipe wall at $z=372.4 \mathrm{~mm}$ 


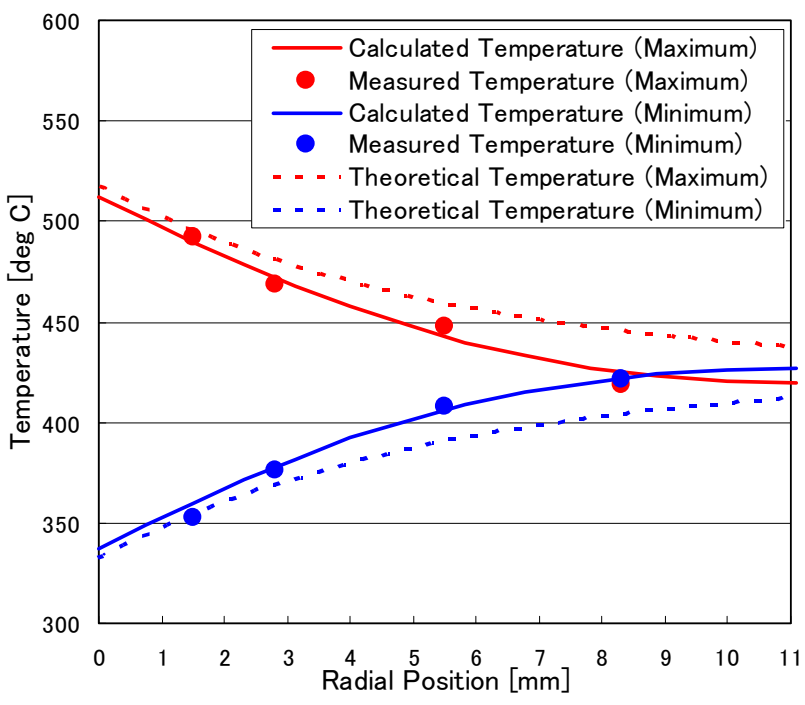

Fig. 11 Radial temperature distribution, DES model

These characteristics were also observed for the k- $\varepsilon$ and SST models. The temperature ranges calculated for the third cycle of fluctuation of fluid and wall temperature are listed in Table 1. Comparison of the numerical simulation ranges with the experiments showed that estimation accuracy was within $11 \%$ range.

Table 1 Temperature fluctuation range at $z=372.4 \mathrm{~mm}[\operatorname{deg} \mathrm{C}]$

\begin{tabular}{|l|r|r|r|r|r|}
\hline $\begin{array}{c}\text { Types of } \\
\text { turbulence model }\end{array}$ & $\begin{array}{c}\text { Fluid } \\
(-1 \mathrm{~mm})\end{array}$ & $\begin{array}{l}\text { Pipe Wall } \\
(1.5 \mathrm{~mm})\end{array}$ & $\begin{array}{l}\text { Pipe Wall } \\
(2.8 \mathrm{~mm})\end{array}$ & $\begin{array}{l}\text { Pipe Wall } \\
(5.5 \mathrm{~mm})\end{array}$ & $\begin{array}{l}\text { Pipe Wall } \\
(8.3 \mathrm{~mm})\end{array}$ \\
\hline Experimnt & 186.31 & 139.47 & 98.37 & 62.6 & 45.07 \\
\hline DES & 188.75 & 132.1 & 95.55 & 64.91 & 48.57 \\
\hline $\mathrm{k}-\varepsilon$ & 189.03 & 125.18 & 93.26 & 60.92 & 43.38 \\
\hline SST & 189.37 & 125.02 & 93.24 & 60.96 & 43.41 \\
\hline
\end{tabular}

\subsection{Thermal stress}

\subsubsection{Thermal stress distribution}

The temperature distributions of the pipe wall as simulated with CFD analysis were converted into ABAQUS input data via IMAT-F, to calculate thermal stress. The instantaneous stress distributions calculated with the simulated result of the DES model are shown in Fig. 12, where (a) shows hoop stress $\sigma_{\theta}$ and (b) shows axial stress $\sigma_{z}$ when tensile stress reached maximum at the beginning of the third cycle $t=41 \mathrm{~s}$. The maximum or minimum stress was observed at the thicker area of pipe wall, since the temperature gradient along the radial direction was steeper. At the inner surface, the hoop and axial stress were tensile; at the outer surface, these stresses were compressive. 


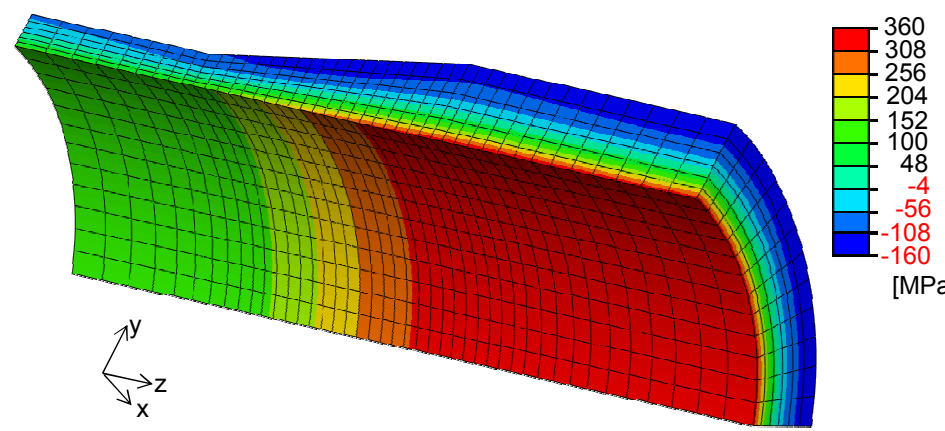

(a)Hoop stress $\sigma_{\theta}$

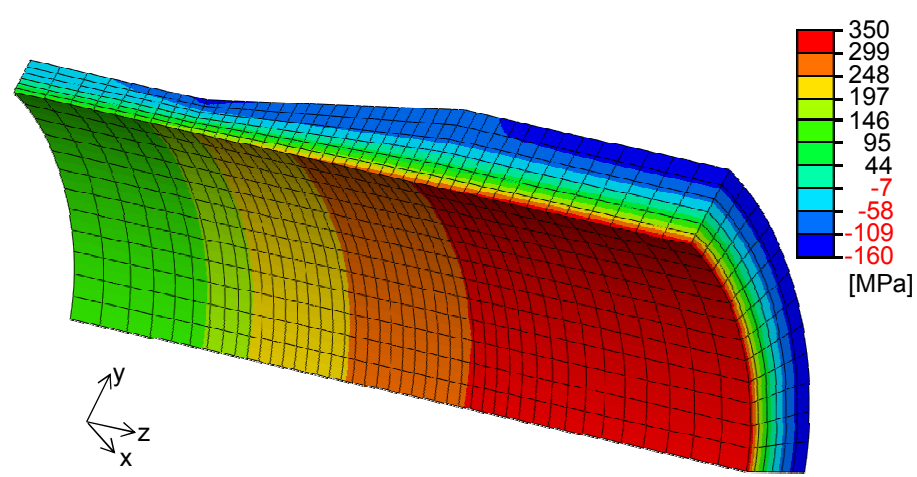

(b)Axial stress $\sigma_{z}$

Fig. 12 Instantaneous stress distribution (close-up of tapered region)

\subsubsection{Thermal stress fluctuation}

Two cross lines were set at the tapered area to evaluate stress, as shown in Fig. 13; the thermal stress fluctuations at the inner surface of these lines, as calculated with results of the DES model, are shown in Fig. 14. Hoop stress $\sigma_{\theta}$ and axial stress $\sigma_{z}$ were dominant in comparison with other stresses, which were nearly 0 . At line A, axial stress was greater than hoop stress, but at line B, the two were nearly equal. Since there was a thicker region downstream of line A, axial deformation was restrained there, causing greater stress than seen in hoop stress. On line B, in contrast, there was no structural feature that restrained axial deformation, which fact resulted in near equality of axial and hoop stresses.

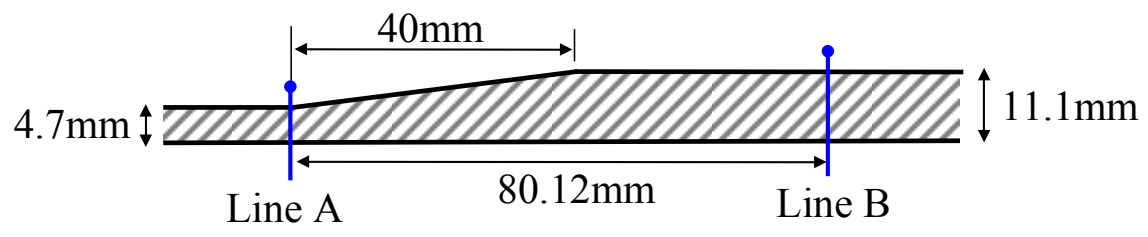

Fig. 13 Cross lines for evaluating thermal stress 


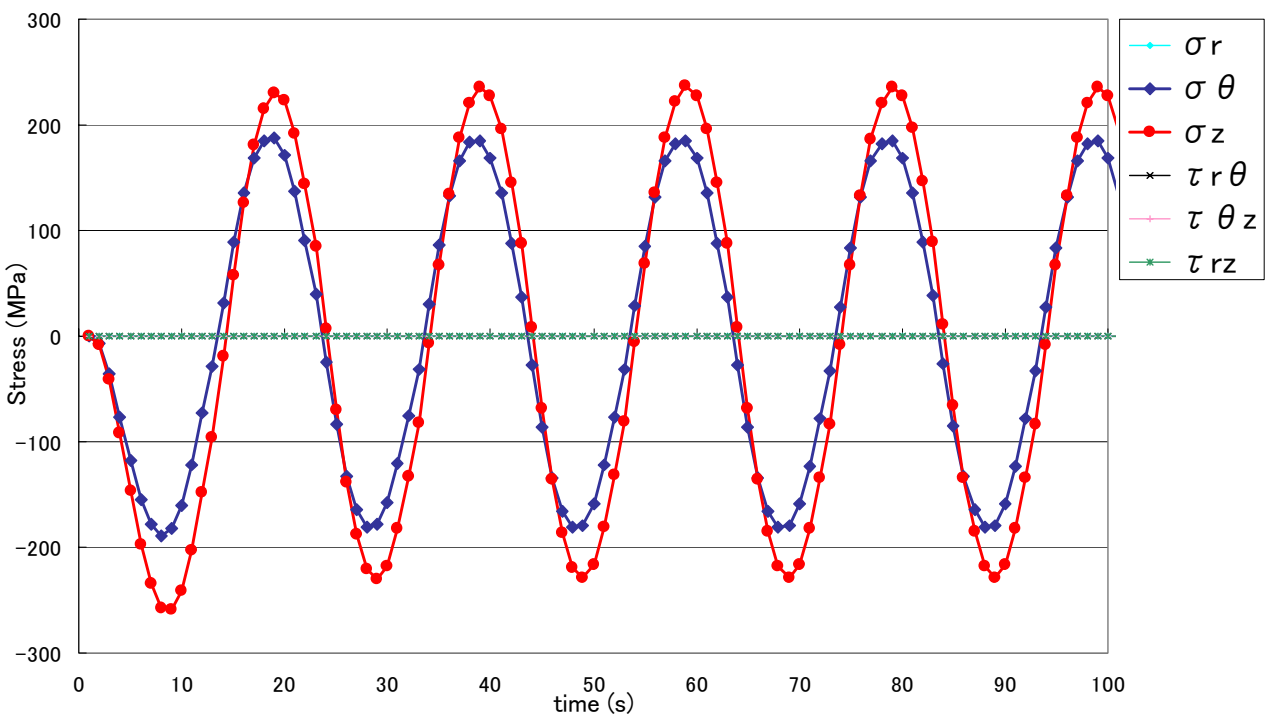

(a) At line $\mathrm{A}$

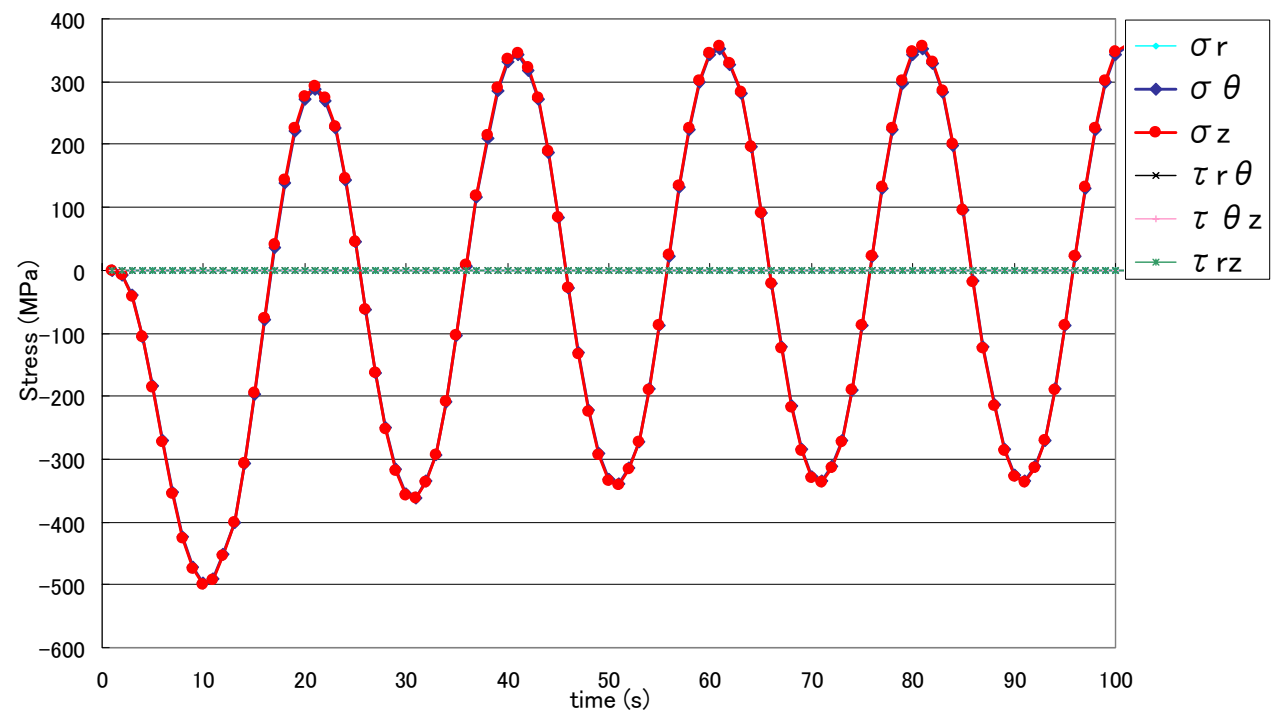

(b) At line B

Fig. 14 Thermal stress fluctuations at each cross lines

After three cycles from the beginning, the stress fluctuation could be assumed a sinusoidal wave; in other words, it had reached a quasi-steady state. The thermal stress amplitude $\Delta \sigma_{\max }$, as calculated from the stress range of the third cycle for each turbulence model, is given in Fig. 15, which shows the Tresca stress of the thermal stress difference as defined by Formula (3).

$$
\Delta \sigma_{\text {max }}=\left|\Delta \sigma_{1}-\Delta \sigma_{3}\right| \quad\left(\sigma_{1} \geq \sigma_{2} \geq \sigma_{3}\right)
$$

where $\Delta \sigma_{1}, \Delta \sigma_{2}, \Delta \sigma_{3}$ are the primary stresses difference. Fig. 15 shows that the simulation result with the DES model had the largest stress amplitude. This is consistent with the fact that the temperature fluctuation of the DES model was largest at the inner surface, as shown in Table 1. The difference between the turbulence models was relatively small and within $2 \%$ of overall fluctuation. 


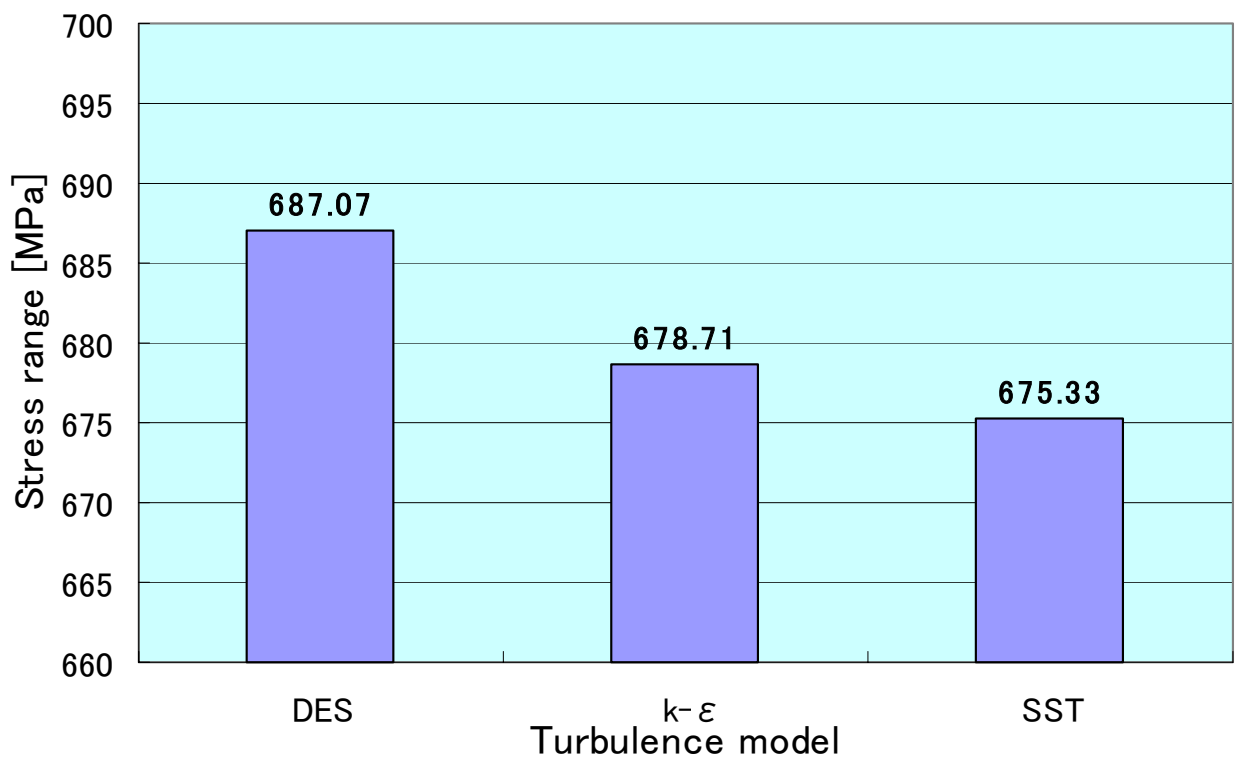

Fig. 15 Thermal stress range for each turbulence model

\subsection{Fatigue life}

The fatigue life for the experiment was estimated in accordance with the design and construction code for fast breeding reactors established by JSME ${ }^{(13)}$. The predicted fatigue life for each turbulence model and result of the experiment is listed in Table 2, which also lists the relevant values used to calculate fatigue life. Since there are safety margins included in the design fatigue curve for 2 in strain range and 20 in fatigue life, the best-fit curves $^{(14)}$ excluding those safety margins were also considered during prediction. The prediction with design fatigue curve was approximately 40 times shorter than the experimental results. As for the fatigue life calculated with the best-fit curve, the prediction with the $\mathrm{k}-\varepsilon$ model was the value closest to the experimental result. As for the difference between the turbulence models, the result calculated with the SST model predicted the longest fatigue life, its difference being within $29 \%$ in comparison with the shortest fatigue life, calculated with the DES model. Even though there is only a $1.9 \%$ difference in thermal stress range $S_{n}{ }^{*}$ as shown in Table 2, the resulting fatigue life was over ten times the thermal stress range. This indicates as regards high cycle fatigue, a small difference in stress (strain) will greatly affect the predicted fatigue life.

Table 2 Predicted fatigue life for each turbulence model [cycles]

\begin{tabular}{|c|c|c|c|c|}
\hline & DES & $\mathrm{k}-\varepsilon$ & SST & Experiment \\
\hline $\begin{array}{l}\text { Stress range } S_{n}{ }^{*}[\mathrm{MPa}] \\
\text { (Without peak stress) }\end{array}$ & 328.69 & 324.25 & 322.68 & \multirow{5}{*}{. } \\
\hline Stress concentration factor $K[-]$ & 0.7482 & 0.7389 & 0.7498 & \\
\hline Strain concentration factor $K_{\varepsilon}[-]$ & 0.760 & 0.741 & 0.736 & \\
\hline Nominal Strain range $\varepsilon_{n}[\mathrm{~mm} / \mathrm{mm}]$ & 0.002107 & 0.002079 & 0.002068 & \\
\hline Strain range $\varepsilon_{t}[\mathrm{~mm} / \mathrm{mm}]$ & 0.002677 & 0.002616 & 0.002591 & \\
\hline Fatigue life(Design curve) & 3,880 & 4,403 & 4,689 & \multirow{2}{*}{157,150} \\
\hline Fatigue life(Best-fit curve) & 128,771 & 153,494 & 164,962 & \\
\hline
\end{tabular}

\section{Conclusions}

This paper describes the development and validation of the IMAT-F system, the 
Integrated Methodology for Assessing Thermal Fatigue, as applied to SPECTRA conducted by JAEA. The validation results derived the following conclusions:

1) IMAT-F is capable of simulating temperature fluctuation of fluid and pipe wall, calculating thermal stress fluctuation and estimating fatigue life. Fatigue life was estimated by applying the temperature fluctuation of SPECTRA. It was confirmed that IMAT-F can integrate CFD analysis output and FEA input via data conversion.

2) Temperature fluctuation range accuracy was all within $11 \%$ in comparison with SPECTRA measurement. This indicates that temperature fluctuation of fluid and pipe wall in the SPECTRA experiment are simulated with considerable accuracy for all turbulence models.

3) Differences in temperature fluctuation range among the three turbulence models were all within $12 \%$. Turbulence model type had relatively little influence on pipe wall fluctuation in the SPECTRA experiment.

4) The predicted fatigue life with best-fit curve had a maximum of $18 \%$ uncertainty in comparison with the experimental results. The difference in fatigue life among the three turbulence models was $29 \%$.

\section{Acknowledgements}

The authors would like to thank Mr. Tohru Sasaki, Kawasaki Plant Systems, Ltd., for his contribution in developing the IMAT-F system, and Michio Murase for his advice.

\section{References}

(1) The Japan Society of Mechanical Engineers, Guideline for evaluation of High-Cycle Thermal Fatigue of a Pipe (in Japanese), (2003), JSME S017.

(2) T. Muramatsu, N. Kasahara, M. Kikuchi, M. Nishimura and H. Kamide, Current Status and Future Plan for Thermal Striping Investigations at JNC (in Japanese), (2000), JNC TN-9400 2000-010.

(3) C. Péniguel, M. Sakiz, S. Benhamadouche, J.M. Stephan and C. Vindeirinho, Presentation of a numerical 3D approach to tackle thermal striping in a PWR nuclear T-junction, Proceedings of ASME PVP Division Conference 2003, (2003-7), PVP2003-1291

(4) S. Chapuliot, C. Gourdin, T. Payen, J.P. Magnaud and A. Monavan, Hydro-thermal-mechanical analysis of thermal fatigue in a mixing tee, Proceedings of Third International Conference Fatigue of Reactor Components, (2004-10), Session No. 2a-1.

(5) S. Hasebe, S. Kobayashi, M. Ando and N. Kasahara, Experimental Study on Properties of High Cycle Thermal Fatigue -Outline and Test Plan of High Cycle Fatigue Test Equipment on Sodium- (in Japanese), (2003), JNC TN9400 2003-004.

(6) S. Hasebe, S. Kobayashi, H. Tanaka, K. Ibaraki and H. Fukasaku, Experimental Study on Properties of High Cycle Thermal Fatigue (3) -Results of sinusoidal temperature fluctuation test at 20 seconds cycle (in Japanese), (2004), JNC TN9400 2004-034.

(7) ANSYS, Inc., CFX-10 Users Manual, (2005).

(8) S. V. Patankar, Numerical Heat Transfer and Fluid Flow, (1980), Hemisphere Publishing.

(9) ABAQUS Inc., ABAQUS ver.6.5 Users Manual, (2004).

(10) The Japan Society of Mechanical Engineers, Codes for Nuclear Power Generation Facilities -Rules on Design and Construction for Nuclear Power Plants- $<$ Part 1 Code for Light Water Reactors> (in Japanese), (2005), JSME S NC1-2005.

(11) American Society of Mechanical Engineers, 2004 ASME Boiler \& Pressure Vessel Code Sec.II Part D(Metric), (2004).

(12) The Japan Society of Mechanical Engineers, Den-netsu Kougaku Siryou Revised 4th 
edition (in Japanese), (1986), p.14.

(13) The Japan Society of Mechanical Engineers, Codes for Nuclear Power Generation Facilities -Rules on Design and Construction for Nuclear Power Plants- $<$ Part 2 Fast Breeding Reactors> (in Japanese), (2005), JSME S NC2-2005.

(14) Japan Atomic Energy Agency, Kaisetsu Kousoku-genkeiro-kouon-kouzou-sekkei-housin Zairyou-kyoudo-kijyuntou (in Japanese), (1984), PNC TN241 84-10 p.141. 